EPJ Web of Conferences 64, 01001 (2014)

DOI: $10.1051 /$ epjconf/ 20146401001

(C) Owned by the authors, published by EDP Sciences, 2014

\title{
Theory of Disk Accretion onto Magnetic Stars
}

\author{
Dong Lai ${ }^{1, a}$ \\ ${ }^{1}$ Department of Astronomy, Cornell University, Ithaca, NY 14853, USA
}

\begin{abstract}
Disk accretion onto magnetic stars occurs in a variety of systems, including accreting neutron stars (with both high and low magnetic fields), white dwarfs, and protostars. We review some of the key physical processes in magnetosphere-disk interaction, highlighting the theoretical uncertainties. We also discuss some applications to the observations of accreting neutron star and protostellar systems, as well as possible connections to protoplanetary disks and exoplanets.
\end{abstract}

\section{Introduction}

Disk accretion onto magnetic central objects occurs in a variety of astrophysical contexts, ranging from classical T Tauri stars (e.g., Bouvier et al. 2007) and cataclysmic variables (intermediate polars; e.g. Warner 2004), to accretion-powered X-ray pulsars (e.g. Lewin \& van der Klis 2006). Such accretion has been studied extensively since the discovery of high-mass accreting X-ray pulsars in the 1970s (e.g., Pringle \& Rees 1972). The basic picture of disk-magnetosphere interaction is well known. The stellar magnetic field disrupts the accretion flow at the magnetospheric boundary and funnels the plasma onto the polar caps of the star or ejects it to infinity (e.g., Pringle \& Rees 1972). The magnetosphere boundary is located where the magnetic and plasma stresses balance,

$$
r_{m}=\xi\left(\frac{\mu^{4}}{G M \dot{M}^{2}}\right)^{1 / 7},
$$

where $M$ and $\mu$ are the mass and magnetic moment of the central object, $\dot{M}$ is the mass accretion rate and $\xi$ is a dimensionless constant of order 0.5-1. Roughly speaking, the funnel flow occurs when $r_{m}$ is less than the corotation radius $r_{c}$ (where the disk rotates at the same rate as the star). For $r_{m} \gtrsim r_{c}$, centrifugal forces may lead to ejection of the accreting matter ("propeller" effect; Illarionov $\&$ Sunyaev 1975).

However, beyond this simple "Astro-101" picture (with the estimate of $r_{m}$ given above), the situations are far more complicated. Over the last several decades, numerous theoretical studies have been devoted to understanding the interaction between accretion disks and magnetized stars. Many different models have been developed. Some examples are (see, e.g., Ghosh \& Lamb 1979; Aly 1980; Lipunov \& Shakura 1980; Anzer \& Börner 1980,1983; Arons 1987,1993; Wang 1987,1995; Aly \& Kuijpers 1990; Spruit \& Taam 1990,1993; Shu et al. 1994,2000; van Ballegooijen 1994; Lovelace et al. 1995,1999; Li et al. 1996; Wickramasinge \& Rüdiger 1996; Campbell 1997; Lai 1998,1999;

\footnotetext{
a e-mail: dong@astro.cornell.edu
} 


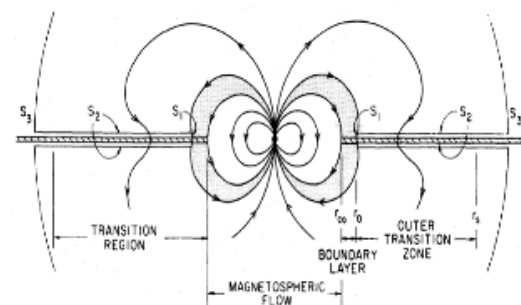

Ghosh \& Lamb 1979

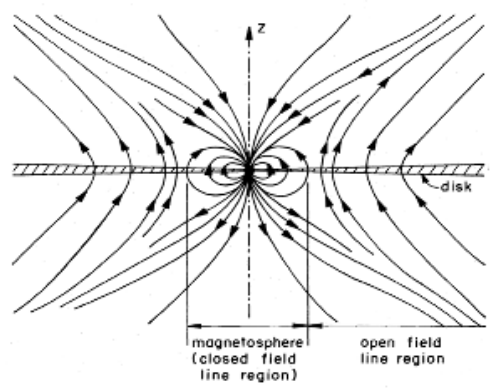

Lovelace et al. 1995

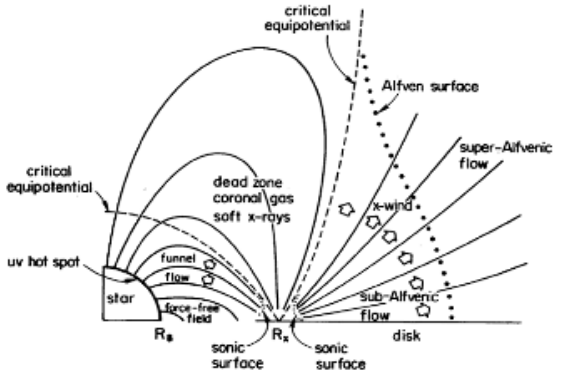

Shu et al. 1994

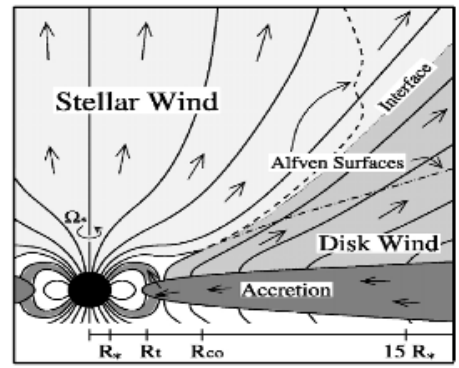

Matt \& Pudritz 2005

Figure 1. Different theoretical models of magnetosphere-disk interaction (see text for discussion).

Terquem \& Papaloizou 2000; Shirakawa \& Lai 2002a,b; Pfeiffer \& Lai 2004; Uzdensky et al. 2002, Uzdensky 2004; Matt \& Pudritz 2005; D’Angelo \& Spruit 2010):

- Ghosh \& Lamb (1979) assumed that the stellar fields invade the disk over a large range of radii. Differential rotation between the star and the disk will cause the field lines to wind up. They argued that a steady state can be reached, where the winding-up of the field lines is balanced by dissipation, so that the field lines slip across the disk. A problem with the GL picture is that in order to avoid too much winding, they need a very large (and unrealistic) magnetic diffusivity.

- In fact, the accretion disk is a very good conductor. So it is possible that the stellar magnetic field cannot penetrate the disk except near the magnetosphere boundary. This kind of models were first explored by Arons, McKee \& Pudritz (see Arons 1986). The field lines are strongly pinched and a centrifugally driven wind may come out from the magnetosphere boundary. These ideas were worked out and expanded in more details in the so-called x-wind model of Shu et al. (1994), which has a rich set of phenomenology and applications.

- Lovelace et al. (1995) considered a model where the field lines connecting the star and disk becomes open because of the winding-up. So there is an inner magnetosphere corotating with the star but also an open region where wind may come out.

- Matt \& Pudritz (2005) developed a model where the possible role of stellar wind driven by accretion was emphasized..

In parallel to these theoretical studies, since 1990s there have been many numerical simulations, with increasing sophistication, on this problem (Hayashi et al. 1996,2000; Miller \& Stone 1997; Goodson et al. 1997; Fendt \& Elstner 2000; Matt et al. 2002; Romanova et al. 2003,2009). These 
simulations are playing an important role in elucidating the physics of magnetosphere-disk interaction in various astrophysical situations (see papers by Romanova and by Zanni in this volume).

In this paper, we will not discuss any particular models or simulations, which are covered by others. Instead, we will focus on several uncertain issues in the theory of magnetosphere-disk interaction, and will also discuss their possible applications and relevance in various astrophysical situations.

\section{Magnetospheric Boundary and Disk Inner Radius}

Let us first consider the problem of dipole magnetic field of a star being invaded by a conducting disk (Aly 1980). Note that an accretion disk is indeed a good conductor. MRI-driven turbulence gives rise to both viscosity $v$ and magnetic diffusivity $\eta$, with the ratio (called magnetic Prantl number) $v / \eta$ of order a few (see the simulation by Lesur \& Longaretti 2009). With the $\alpha$-parameterization, $\eta \sim v=\alpha H c_{s}$, the diffusion time of magnetic field across the disk is $t_{\text {diff }} \sim H^{2} / \eta \sim 1 /(\alpha \Omega)$ (where $\Omega$ is the disk rotation velocity), much longer than the disk dynamical time.

For a perfectly conducting disk/plate (with inner radius $r_{\text {in }}$ surrounding a dipole, the exact solution (Aly 1980) shows that the vertical field at the disk inner edge is $B_{z}\left(r_{\text {in }}\right) \simeq B_{\star}\left(r_{\text {in }}\right)\left(r_{\text {in }} / H\right)^{1 / 2}$, where $B_{\star}\left(r_{\text {in }}\right) \sim \mu / r_{\text {in }}^{3}$ is the dipole field at $r=r_{\text {in }}$ when the disk is absent.

Now, at the inner edge of the disk, various instabilities (including magnetic Kelvin-Helmholtz and Rayleigh-Taylor, and reconnection) can develop, leading to the formation of a boundary layer. The stellar dipole field penetrates this magnetospheric boundary layer and gets twisted to produce $B_{\phi \pm}=\mp \zeta B_{z}$, with $\zeta \sim 1$ (the upper/lower sign specifies the field above/below the disk). In the boundary layer $\left(r_{m}<r<r_{m}+\Delta r_{m}\right)$, the plasma angular velocity transitions from the Keplerian rate $\Omega_{K}$ to the stellar spin rate $\Omega_{s}$ as a result of the magnetic torque:

$$
r^{2} B_{z} B_{\phi+}=-\dot{M} d\left(r^{2} \Omega\right) / d r
$$

For $\Omega_{K}\left(r_{m}\right) \gg \Omega_{s}$, we have $d\left(r^{2} \Omega\right) / d r \sim r^{2} \Omega_{K} / \Delta r_{m}$, and

$$
r_{m} \simeq\left(\frac{\zeta \Delta r_{m}}{H}\right)^{2 / 7}\left(\frac{\mu^{4}}{G M \dot{M}^{2}}\right)^{1 / 7} .
$$

Except for the first factor on the right-hand side, this is the usual expression of magnetosphere radius for spherical accretion.

Note that equation (3) applies to the case of $\Omega_{K}\left(r_{m}\right) \gg \Omega_{s}$, or equivalently, the corotation radius $r_{c}$ (where $\Omega_{K}$ equals $\Omega_{s}$ ), is much larger than $r_{m}$ ). For $\Omega_{s} \gg \Omega_{K}\left(r_{m}\right)$ (or $r_{c}<r_{m}$ ), we have

$$
r_{m} \simeq\left(\frac{\zeta \Delta r_{m}}{H}\right)^{1 / 5}\left(\frac{\mu^{2}}{\dot{M} \Omega_{s}}\right)^{1 / 5} .
$$

In practice, the difference between equations (3) and (4) is rather small, given the other uncertainties in the problem.

The next question one can ask is whether $r_{m}$ (as determined above) equals $r_{\text {in }}$, the inner radius of the disk. There are two issues to keep in mind:

(i) In principle, a disk-like structure, corotating with the star at $\Omega_{s}$, could exist inside $r_{m}$. Note that this structure does not Keplerian rotation, but is part of the corotating magnetosphere. This "disk" plasma can drift inward by interchange instability (Spruit \& Taam 1990). At some point (depending on the field geometry), this "disk" can be disrupted and the plasma flows along the field line to the magnetic polar cap of the star. 


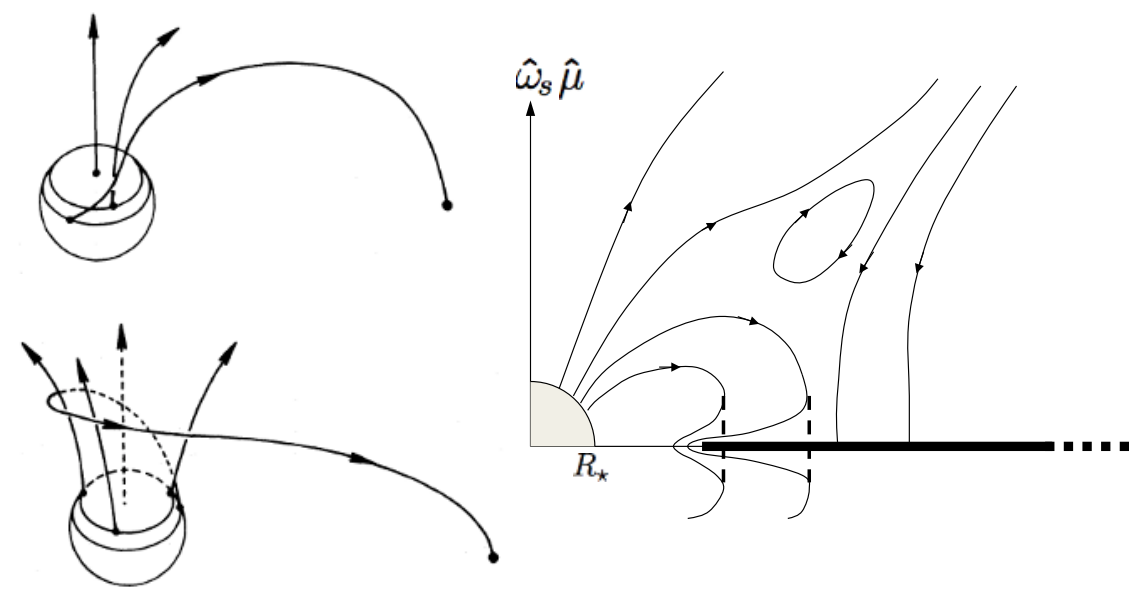

Figure 2. Left: Magnetic field lines linking the star and disk are twisted by differential rotation (Aly \& Kuijpers 1990). Right: Quasi-cyclic behavior (see text).

(ii) More importantly, the expression for $r_{m}$ given above assumes steady state. In another word, $\dot{M}$ in the above expression is the local value at $r_{m}$. It is possible that if mass accumulates at the boundary (e.g., by the centrifugal barrier; see below), the local $\dot{M}$ is larger than the mass supply rate $\left(\dot{M}_{\text {supply }}\right)$ at larger distance. This may lead to episodic accretion. For example, when $\dot{M}$ is small, $r_{m}$ will be larger than $r_{c}$, while $r_{\text {in }}$ can be much less than $r_{m}$. Such episodic accretion scenario has been explored by Spruit \& Taam (1993) and D'Angelo \& Spruit (2010,2011,2012). One issue concerning such a scenario is the possible non-axisymmetric instabilities that may develop, allowing the accumulated mass to fall rapidly through the magnetosphere onto the star.

\section{Star-Disk Linkage and Magnetic Field Inflation}

When the stellar field lines penetrate some region of the disk, they provide a linkage between the star and the disk. These field lines are twisted by differential rotation between the stellar rotation $\Omega_{s}$ and the disk rotation $\Omega(r)$, generating toroidal field. In steady state - if it can be reached, the twisting is balanced by dissipation across the disk. This gives the toroidal field just above the disk, $B_{\phi+} \sim B_{z}\left(\Omega-\Omega_{s}\right) H^{2} / \eta$. With $\eta \sim \alpha H c_{s}$, we find $B_{\phi+} / B_{z} \simeq\left(\Omega-\Omega_{s}\right) /(\alpha \Omega)$. Obviously, except for a narrow region around the corotation radius $r_{c}$, the toroidal field is much stronger than the poloidal field.

However, when the toroidal field becomes comparable to the poloidal field, the flux tube connecting the star and the disk will start expanding. This field inflation is driven by the pressure associated with the toroidal field. As the fields open up, the star-disk linkage is broken. Such field-opening behavior has been well-established through theoretical studies and numerical simulations in the contexts of solar flares and accretion disks (e.g., Aly 1985; Aly \& Kuijpers 1990; van Ballegooijen 1994; Lynden-Bell \& Boily 1994; Lovelace et al. 1995; Uzdensky et al. 2002; see Fig. 2). Thus, the maximum toroidal twist is $\left|B_{\phi+} / B_{z}\right|_{\max } \sim 1$. 

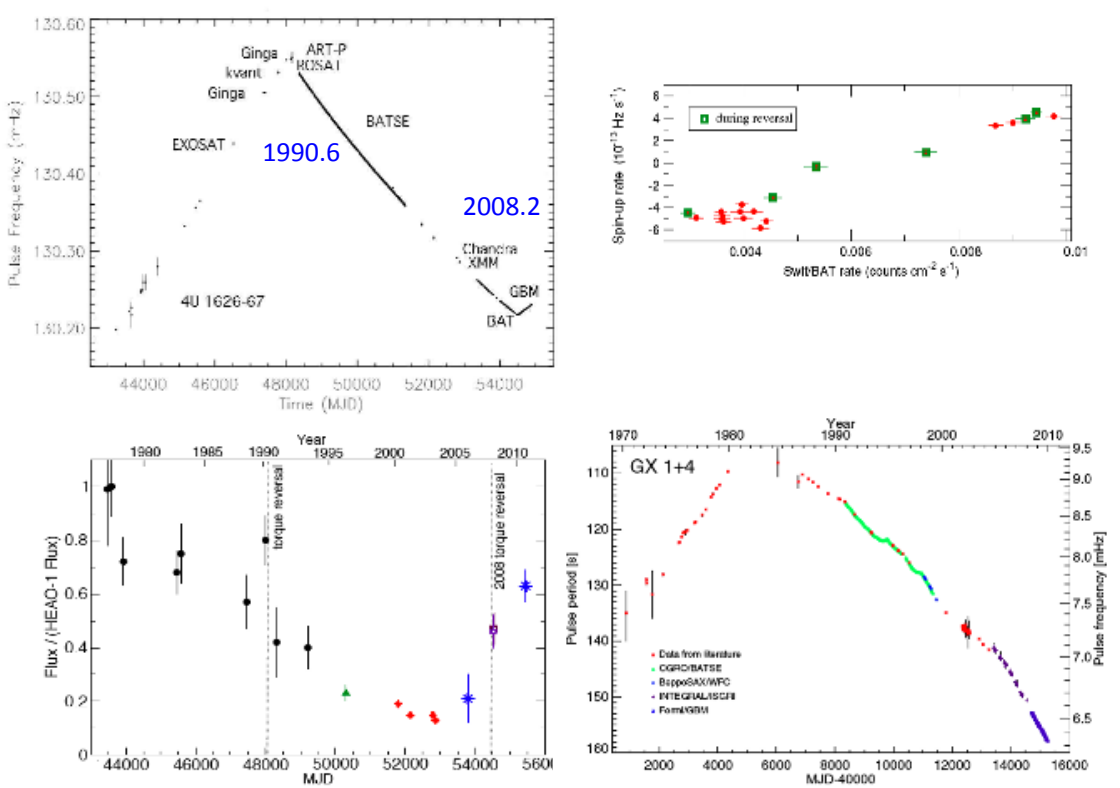

Figure 3. Left panels and the right-top panel: Spin evolution of 4U1626-67 and its correlation with the x-ray flux (see Camero-Arranz et al. 2010,2012). Right-bottom panel: Spin evolution of GX 1+4 (see Chakrabarty et al. 1997; Gonzales-Galan et al. 2012).

Given this constraint on the toroidal twist, steady-state disk-star linkage is possible only very near corotation $\left(\Delta r / r_{\mathrm{co}} \sim \alpha\right)$. In general, we should expect a quasi-cyclic behavior, involving several stages: (1) The stellar field penetrates the inner region of the disk; (2) The linked field lines are twisted; (3) The resulting toroidal fields drive field inflation; (4) Reconnection of the inflated field restores the linkage. The whole cycle then repeats (see Aly \& Juijpers 1990; Uzdensky et al. 2002).

Given that such a quasi-cyclic behavior is inevitable, questions arise as to how this behavior may manifest observationally. In particular, is there any connection with QPOs in low-mass X-ray binaries (see van der Klis 2005 for a review) and other systems? In particular, QPOs with frequencies around $\mathrm{kHz}$ have been observed in a number of accreting millisecond pulsars. The quasi-cyclic timescale is comparable to the $\mathrm{kHz}$ QPO timescales, but exactly how these QPOs are produced remains unclear.

The quasi-cyclic state may also give rise to episodic outflows and winds, to be discussed later.

\section{Torque on the Star, Propeller, Spin Equilibrium}

Although a precise steady-state of the star-disk linkage cannot be achieved, one can nevertheless ask the question: On average (i.e., over many dynamical times), what is the width $(\delta r)$ of the magnetically linked region in the disk? The reason that this is of interest is that the width $\delta r$ directly affects the long-term (secular) torque on the star and therefore its equilibrium rotation rate. 
In general, the torque in the star has two contributions, one associated with mass accretion and another related to star-disk coupling:

$$
\frac{d J_{\star}}{d t}=\dot{M}_{\mathrm{acc}} r_{m}^{2} \Omega\left(r_{m}\right)+T_{m},
$$

where $\dot{M}_{\text {acc }}$ is the mass accretion rate onto the star, which, in general, could be different from $\dot{M}_{\infty}$ or even $\dot{M}$ (see above). The magnetic torque is

$$
T_{m}=\int_{r_{m}}^{r_{m}+\Delta r} d r 2 \pi r\left(-r \frac{B_{z} B_{\phi+}}{2 \pi}\right) \simeq \zeta\left(r^{2} B_{z}^{2}\right)_{r_{m}} \Delta r,
$$

where in the second equality we have assumed $\Delta r$ is small and used $B_{\phi+}=-\zeta B_{z}$. Using $B_{z} \simeq$ $\left(\mu / r^{3}\right)(r / H)^{1 / 2}$, we find

$$
T_{m} \simeq\left(\frac{\zeta \Delta r}{H}\right) \frac{\mu^{2}}{r_{m}^{3}}
$$

Using equation (3) and assuming $\delta r$ is the same as the width of the boundary layer $\delta r_{m}$, we find

$$
\left|T_{m}\right| \simeq \dot{M} \sqrt{G M r_{m}}
$$

Thus when $\dot{M} \simeq \dot{M}_{\text {acc }},\left|T_{m}\right|$ os of the same order of magnitude as the accretion torque. Note, however, that $T_{m}$ can have either positive or negative sign: While we expect $|\zeta| \sim 1$, the sign of $\zeta$ depends on the location of the interaction zone relative to the corotation radius $r_{c}$. For $r \simeq r_{m}<r_{c}$, we have $\zeta>0$ and the magnetic torque on the star is positive, while for $r_{m}>r_{c}$, we have $T_{m}<0$.

The torque on the star is affected by the propeller effect. When the accreting plasma penetrates the magnetosphere and gets attached to the stellar field lines, it corotates with the star. If $r_{m} \Omega_{s}$ exceeds the escape speed $v_{\text {esc }}\left(r_{m}\right)=\left(2 G M / r_{m}\right)^{1 / 2}$, or equivalently $r_{m}>2^{1 / 3} r_{c}$, the plasma may be ejected due to centrifugal force. The torque on the star should be modified to

$$
\frac{d J_{\star}}{d t}=\dot{M}_{\mathrm{acc}} r_{m}^{2} \Omega\left(r_{m}\right)+T_{m}-\dot{M}_{\mathrm{eject}} r_{m}^{2} \Omega_{s}
$$

where $\dot{M}_{\text {eject }}$ is the mass ejection rate. In steady-state, we may expect $\dot{M}_{\text {acc }}+\dot{M}_{\text {eject }}=\dot{M}$. Precisely how much mass is ejected would depends on details of dissipation/heating near the magnetosphere boundary, magnetic field topology, etc., If the plasma is not ejected, it may accumulate near the magnetosphere boundary, forming a dead disk (D'Angelo \& Spruit 2010,2012). Is there any accretion onto the star through interchange instabilities? Determining $\dot{M}_{\text {acc }}$ and $\dot{M}_{\text {eject }}$ in the canonical propeller regime is an important problem.

Of course, the reason we are interested in the torque on the star is because we want to know what physics determines the equilibrium spin of accreting magnetic stars. This is relevant to young stars ( T tauri stars), accreting millisecond pulsars as well as long-period pulsars. In the standard picture, already developed in the 1970s and 1980s, the equilibrium rotation equals, to within a factor of two, the Keplerian rotation rate at $r_{m}$. That is, equilibrium is reached when $r_{c} \simeq r_{m}$. Although there may be various factors (accretion torque, magnetic torque, propeller, wind/outflows, etc) that complete to drive the star's rotation, all these generally lead to the similar equilibrium condition $\left(r_{c} \sim r_{m}\right)$.

More constraining to the theory of magnetic star-disk interaction is the spinup/spindown behavior of accreting x-ray pulsars. Many x-ray pulsars have been observed to exhibit changing spinup and spindown behaviors over timescale of years (Bildsten et al. 1997). Figure 3 shows two particularly striking examples: 4U1626-67 is an accreting pulsar with spin period $7.66 \mathrm{~s}$. The clean spinup 
before 1990.6 was followed by a clean spindown, and another spinup phase starting 2008.2. The spindown/spinup transition lasted 150 days. The spinup rate $\dot{\Omega}_{s}$ is positively correlated with the X-ray flux (Camero-Arranz et al. 2010,2012). In GX 1+4, it was found that during the spindown phase, the magnitude of the spindown torque is positively correlated with the X-ray flux, $-\dot{\Omega}_{s} \propto F_{x}^{0.030 \pm 0.07}$ (Chakrabarty et al. 1997; Gonzalez-Galan et al. 2012). This latter correlation is difficult to explain. In fact, it directly contradicts the prediction of the original Ghosh-Lamb model. Perhaps some variations of the models based on variable $\dot{M}_{\text {acc }}$ and $\dot{M}_{\text {eject }}$ can do the job, but exactly how is not clear (see Locsei \& Melatos 2004; Perna, Bozzo \& Stella 2006; Dai \& Li 2006 for related works). Understanding this spindown/spinup behavior and its correlation with the accretion rate remains an outstanding unsolved problem.

\section{Outflows}

Outflows is a natural consequence of magnetosphere-disk interaction. We have already touched upon two effects that may give rise to outflows.

(1) The quasi-cyclic star-disk linkage (see Section 3) and associated field inflation inevitably lead to ejection of plasma from the magnetosphere boundary. This episodic outflows have been seen in simulations (see Romanova et al. 2009; Zanni \& Ferreira 2013), but may also be related to the Xwind model of Shu et al. (although the "canonical" X-wind model was developed for the case when the star has already reached spin equilibrium, thus $r_{m} \simeq r_{c}$, and the wind is steady).

(2) In the propeller regime, plasma may be ejected from the magnetosphere. Such outflow is distinct from the boundary outflow discussed in (1). It maybe more more collimated and has a faster speed (see Lii et al. 2013 for recent simulations).

In addition to the above two outflow launching mechanisms, there are two other possibilities:

(3) Accretion-induced stellar wind (see paper by R. Pudritz).

(4) Outflow from the disk through magneto-centrifugal mechanism a la Blandford-Payne. Perhaps such outflow is less collimated and has a slow velocity.

It is possible that some of these mechanisms are related to each other and therefore not distinguishable in practice (both in simulations and observations). Obviously, it is important to confront all these mechanisms with observations. Outflows/jets from young stellar objects are well known and discussed elsewhere. But there are also observed in accreting neutron stars in binaries. Perhaps the famous is the relativistic jet from Circinus X-1 (Fender et al. 2004; Armstrong et al. 2013), a bright and highly variable $\mathrm{x}$-ray binary containing a neutron star on an eccentric orbit (17 days) with a normal star companion. It is interesting to note that jets and outflows have been observed in different types of neutron star binaries, including Atolls sources (such as Aql X-1), Z-sources (Sco X-1) and accreting millisecond X-ray pulsars (SAX J1808). It is usually thought that the key difference in these sources lies in their magnetic field strengths, with accreting pulsars having the highest fields $\left(\sim 10^{9} \mathrm{G}\right)$.

It is also of interest to compare the jet phenomenology in black-hole x-ray binaries and neutronstar x-ray binaries. In the case of black hole, compact steady jets are produced in the low-hard state, when the innermost disk is replaced by a geometrically thick flow/corona; episodic/ballistic jets are produced in the transitional state between the low state and the high, thermal state; no jets are produced in the "clean" thermal state. By contrast, for neutron star systems, jets appear to be produced in the soft x-ray thermal (called "banana") states (for example, Ser X-1, 4U1820-30; see Migliari et al. 2011). The same can be said of young stars, which are always in the "thermal disk" state. Perhaps this difference points to the important role of magnetosphere, which is absent in black-hole systems. 


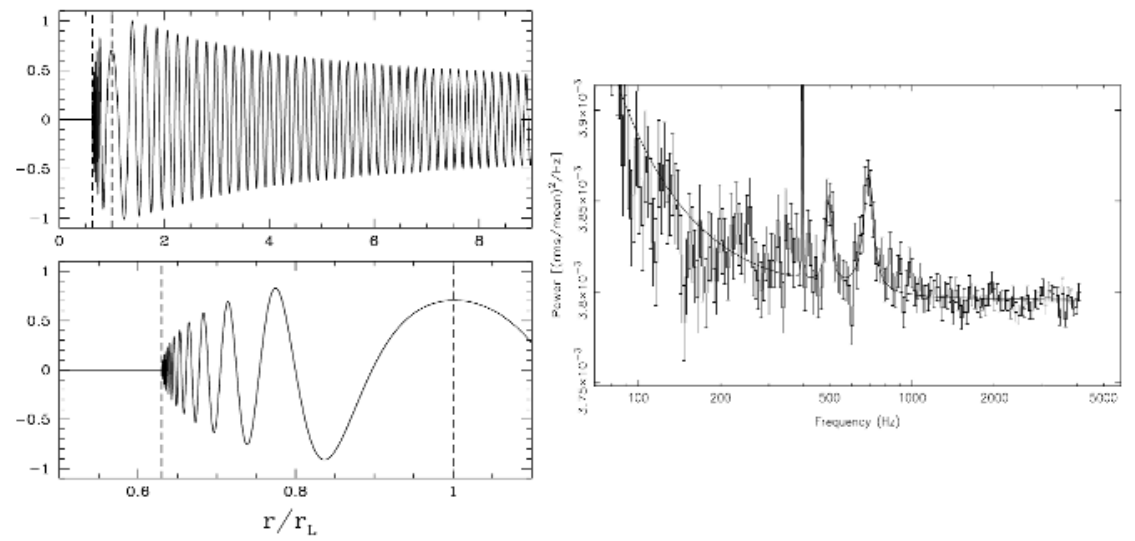

Figure 4. Left panel: Bending waves in the disk excited by magnetic force from a misaligned stellar dipole. The wave is most visible at the Lindblad/vertical resonance (from Lai \& Zhang 2008). Right panel: Power density spectrum of accreting millisecond pulsar SAX J1808.4-3658, showing the spin frequency $v_{s}=401 \mathrm{~Hz}$ and two $\mathrm{kHz}$ QPOs, with $v_{h}-v_{l} \simeq v_{2} / 2$ (from van der Klis 2005).

\section{Misaligned Stellar Fields}

The physics and issues we have discussed so far are applicable regardless of whether the stellar magnetic dipole is aligned or misaligned with the stellar spin axis. In general, the stellar dipole axis is misaligned with the spin (this is required for accreting x-ray pulsars). Are there any new effects? The answer is yes. One obvious effect is that with misaligned magnetic fields, the plasma in the magnetosphere can flow to the polar cap more easily (the so-called "funnel flow"), thereby producing pulsed emission from the stellar surface. With a very aligned stellar field, this may be difficult, and magnetosphere accretion may proceed through instabilities, so that the plasma may land on the stellar surface more in the equatorial region than the polar caps. This obviously will have different observational consequences (see M. Romanova's paper in this proceedings).

Another effect of misaligned stellar dipole is that it can excite non-axisymmetric waves in the disk through magnetic forcing (Lai \& Zhang 2008; see Romanova et al. 2013 for recent simulations). For example, the vertical magnetic force on the disk can be written as

$$
F_{z}(r, \phi, t)=F_{\omega}(r) \exp (i m \phi-i \omega t),
$$

where $m=1$. The forcing frequency $\omega$ can be either $\Omega_{s}$ or $2 \Omega_{s}$. While $\omega=\Omega_{s}$ is obvious, the $2 \Omega_{s}$ component requires some explanation. It arises because while the dipole field at the given point in the disk varies as $\cos \Omega_{s} t$, the induced screening current in the disk (by virtue of its high conductivity) also varies as $\cos \Omega_{s} t$; thus the force has a term proportional $\cos ^{2} \Omega_{s} t$. (Note that this term is present only when the disk is misaligned with the stellar spin; see below). This periodic vertical force will excite bending waves in the disk. These bending waves are launched at the Lindblad/vertical resonance, where the Doppler-shifted forcing frequency $\omega-\Omega(r)$ matches the intrinsic disk vertical epicyclic frequency $\Omega_{z} \simeq \Omega$. Thus, the perturbation is most "visible" at $r=r_{L}$ given by $\Omega\left(r_{L}\right)=\omega / 2=$ $\Omega_{s} / 2, \Omega_{s}$.

These magnetically excited bending waves may have interesting applications to $\mathrm{kHz}$ QPOs observed in accreting millisecond pulsars (van der Klis 2005). In particular, in SAX J1808.4-3658 (with 

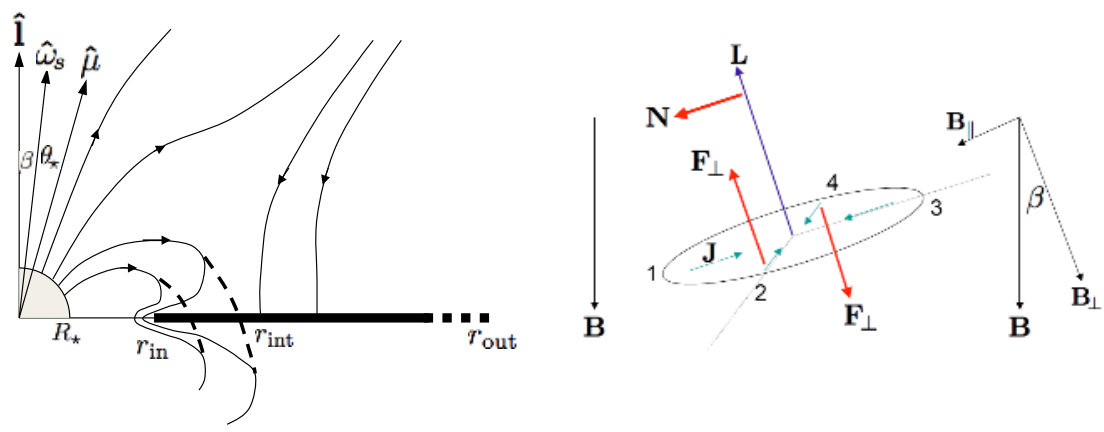

Figure 5. Left panel: A sketch of magnetic field configuration in a star-disk system for nonzero $\beta$ (the angle between the disk axis and the stellar spin axis) and $\theta_{\star}$ (the angle between the stellar dipole axis and the spin axis). Part of the stellar magnetic fields (dashed lines) penetrate the disk in the interaction zone between the disc inner radius $r_{\text {in }}$ and $r_{\text {int }}$ in a cyclic manner, while other field lines are screened out of the disc. The closed field lines are twisted by the differential rotation between the star and the disc, which leads to a magnetic braking torque and a warping torque. The screening current in the disk leads to a precessional torque. Right panel: A toy model for understanding the origin of the warping torque. A tilted rotating metal plate (with angular momentum $\mathbf{L}$ ) in an external magnetic field $\mathbf{B}$ experiences a vertical magnetic force around region 2 and 4 due to the interaction between the induced current $\mathbf{J}$ and the external $\mathbf{B}_{\|}$, resulting in a torque $\mathbf{N}$ which further increases the tilt angle $\beta$.

the spin frequency $v_{s}=401 \mathrm{~Hz}$ ), the two $\mathrm{kHz}$ QPOs frequencies both vary as the source luminosity changes, but the difference frequency $v_{h}-v_{l}$ remains constant and equals $v_{s} / 2$ to within a few Hz. In XTE J1807.4-294 (with $v_{s}=191 \mathrm{~Hz}$ ), the difference $v_{h}-v_{l} \simeq v_{s}$. It seems that these are not pure coincidences. (One should add that for most LMXBs which do not show coherent pulsations, the difference frequency of $\mathrm{kHz}$ QPOs do not exhibit similar correlation with the neutron star spin.) Perhaps the beating of high-frequency QPO $\left(v_{h}\right)$ generated at the magnetosphere (or inside) with the perturbed disk plasma at the Lindblad/vertical resonance can explain these intriguing observations.

\section{Stellar Spin - Disk Misalignment}

In the standard picture magnetic star-disk interaction, it is usually assumed that the stellar spin axis is aligned with the disk axis (the disk normal vector). This seems reasonable since the star may have gained substantial angular momentum from the accreting gas in the disk. However, magnetic interaction between the star and the inner region of the disk may (if not always) change this simple picture (Lai 1999; Lai et al. 2011).

Before being disrupted at $r_{\text {in }}$ by the stellar magnetic field, the disk generally experiences nontrivial magnetic torques from the star. These torques are of two types: (i) A warping torque $\mathbf{N}_{w}$ which acts in a small interaction region $r_{\text {in }}<r<r_{\text {int }}$ (with width $\Delta r$ ), where some of the stellar field lines are linked to the disk. These field lines are twisted by the differential rotation between the star and the disk, generating a toroidal field $\Delta B_{\phi}=\mp \zeta B_{z}^{(s)}$ from the quasi-static vertical field $B_{z}^{(s)}$ threading the disk, where $\zeta \sim 1$ and the upper/lower sign refers to the value above/below the disk plane. Since the toroidal field from the stellar dipole $B_{\phi}^{(\mu)}$ is symmetric with respect to the disk plane, the net toroidal field differs above and below the disk plane, giving rise to a vertical force on the disk. While the mean force (averaging over the azimuthal direction) is zero, the uneven distribution of the force induces a net warping torque which tends to push the orientation of the disk angular momentum $\hat{l}$ away from 
the stellar spin axis $\hat{\boldsymbol{\Omega}}_{s}$. The essential physics of the warping torque can also be understood from the "laboratory" toy model depicted in Fig. 5. (ii) A precession torque $\mathbf{N}_{p}$ which arises from the screening of the azimuthal electric current induced in the highly conducting disk. This results in a difference in the radial component of the net magnetic field above and below the disk plane and therefore in a vertical force on the disk. The resulting precession torque tends to cause $\hat{\boldsymbol{l}}$ to precess around $\hat{\boldsymbol{\Omega}}_{s}$.

The two magnetic torques (per unit area) on the disk can be written as

$$
\mathbf{N}_{w}=-\left(\Sigma r^{2} \Omega\right) \cos \beta \Gamma_{w} \hat{\boldsymbol{l}} \times\left(\hat{\mathbf{\Omega}}_{s} \times \hat{\boldsymbol{l}}\right), \quad \mathbf{N}_{p}=\left(\Sigma r^{2} \Omega\right) \cos \beta \Omega_{p} \hat{\mathbf{\Omega}}_{s} \times \hat{\boldsymbol{l}},
$$

where $\Sigma(r)$ is the surface density, $\Omega(r)$ the rotation rate of the disk, and $\beta(r)$ is the disc tilt angle (the angle between $\hat{\boldsymbol{l}}(r)$ and the spin axis $\hat{\boldsymbol{\Omega}}_{s}$ ). The warping rate and precession angular frequency at radius $r$ are given by

$$
\Gamma_{w}(r)=\frac{\zeta \mu^{2}}{4 \pi r^{7} \Omega(r) \Sigma(r)} \cos ^{2} \theta_{\star}, \quad \Omega_{p}(r)=-\frac{\mu^{2}}{\pi^{2} r^{7} \Omega(r) \Sigma(r) D(r)} \sin ^{2} \theta_{\star},
$$

where $\theta_{\star}$ is the angle between the magnetic dipole axis and the spin axis, and the dimensionless function $D(r)$ is somewhat less than unity.

\subsection{Applications to QPOs/Variabilities}

The magnetically driven warping and precession effects discussed above may be relevant to the lowfrequency (10-50 Hz) QPOs (the so-called horizontal branch oscillations) observed in neutron star LMXBs. Stella \& Vietri (1998) suggested that LFQPOs are associated with the Lense-Thirring precession of the inner accretion disk around the rotating neutron star. If the LFQPO and the $\mathrm{kHz}$ QPOs are generated at the same special radius in the disk, this implies a quadratic relation between the LFQPO frequency and the $\mathrm{kHz}$ QPO frequency, in rough agreement with observations (usually for a certain range of X-ray fluxes). However, in order to have precession, the inner disk must be tilted relative to the stellar spin. The magnetic effects may induce the required disk warp and contribute the the disk precession (Shirakawa \& Lai 2002a, Pfeiffer \& Lai 2004).

Magnetically driven warping and precession may also explain millisecond variabilities in highfield accreting X-ray pulsars (Shirakawa \& Lai 2002b) as well as some photometric variabilities observed in T Tauri stars (such as AA Tau; see Terquem \& Papaloizou 2000; Bouvier et al. 2013).

\subsection{Possible Connection to (Exo)Planetary Systems}

One of the recent surprises in exoplanetary sciences is that many hot Jupiter systems (orbital periods of order a few days) are found to have a misaligned orbital axis relative the spin axis of the host star. Among the several dozens of hot Jupiter systems with measurements the stellar obliquity, about $60 \%$ have an orbital axis aligned (in sky projection) with the stellar spin, while the other systems show a significant spin-orbit misalignment, including some with retrograde orbits.

In our own Solar System, except for Pluto, all planets outside 1 AU lie within $2^{\circ}$ of the ecliptic plane, while the Sun's equatorial plane is inclined by $7^{\circ}$ with respect to the ecliptic. It is not clear whether the difference between $2^{\circ}$ and $7^{\circ}$ is significant or needs an explanation.

The observed spin-orbit misalignment in many hot Jupiter systems indicates that gravitational interactions between multiple planets and/or secular interactions with a distant planet or stellar companion play an important role in shaping the architecture of planetary systems. Nevertheless, there is an alternative possibility: misaligned protostar and protoplanetary disk. Such misaligned disk may explain the $7^{\circ}$ "anomaly" in Solar System and affect the initial condition of planetary formation. 
As discussed above, the inner region of the disk around a magnetic protostar experiences a warping torque and a precessional torque. If we imagine dividing the disk into many rings, and if each ring were allowed to behave independent of each other, it would be driven toward a perpendicular state and precess around the spin axis of the central star. Obviously, real protoplanetary disks do not behave as a collection of non-interacting rings: Hydrodynamic stresses (bending waves and viscosity) provide strong couplings between different rings, and the disk will resist the inner disk warping. Detailed calculations by Foucart \& Lai (2011) showed that for most reasonable stellar/disk parameters, the steady-state disk warp is rather small because of efficient viscous damping or propagation of bending waves. Thus the inner disk direction is approximate the same as the outer disk direction.

What is happening to the stellar spin? Is there a secular change to the stellar spin direction? The answer is maybe. The back-reaction magnetic torque from the disk on the stellar spin can push the stellar spin axis away from alignment. The magnetic misalignment torque on the star is of the same order as the fiducial accretion torque $\dot{M} \sqrt{G M_{\star} r_{\text {in }}}$. Assuming the spin angular momentum $J_{s}=$ $0.2 M_{\star} R_{\star}^{2} \Omega_{s}$, we find the spin evolution time to be

$$
t_{\text {spin }}=(1.25 \mathrm{Myr})\left(\frac{M_{\star}}{1 M_{\odot}}\right)\left(\frac{\dot{M}}{10^{-8} M_{\odot} \mathrm{yr}^{-1}}\right)^{-1}\left(\frac{r_{\mathrm{in}}}{4 R_{\star}}\right)^{-2} \frac{\Omega_{s}}{\Omega\left(r_{\mathrm{in}}\right)} .
$$

Given enough time, the stellar spin axis will evolve towards the perpendicular state and even the retrograde state. Unfortunately, there are other torques acting on the star which counter-act the warping torque. In particular, the accretion torque tends to align the stellar spin axis with the disk. Since the magnetic torque and the accretion torque are of the same order of magnitude, we cannot be make a definitive conclusion. All we can say is that both alignment and misalignment between the stellar spin axis and the disk axis are possible (see Lai, Foucart \& Lin 2011 and Foucart \& Lai 2011 for more details).

Acknowledgments: I thank the organizers for the invitation and support for me to attend the conference on the Physics at the Magnetosphere Boundary in Geneva. This work has been supported in part by NSF grants AST-1008245, 1211061 and NASA grant NNX12AF85G.

\section{References}

[1] Aly, J. J. 1980, A\&A, 86, 192

[2] Aly, J.J. 1985, A\&A, 143, 19

[3] Aly, J. J., \& Kuijpers, J. 1990, A\&A, 227, 473

[4] Anzer, U., \& Börner, G. 1983, A\&A, 122, 73.

[5] Armstrong, R.P. et al. 2013, MNRAS, 433, 1951

[6] Arons, J. 1987, in "The Origin and Evolution of Neutron Stars" (IAU Symp. No. 125), ed. D. J. Helfand \& J.-H. Huang (D. Reidel Pub.: Dordrecht)

[7] Arons, J. 1993, ApJ, 408, 160.

[8] Bildsten, L. et al, ApJS, 113, 367

[9] Bouvier, J., et al. 2007, in Protostars and Planets V, eds. B. Reipurth et al. (Tucson: U. Arizona Press)

[10] Bouvier, J., et al. 2013, A\&A, 557, A77

[11] Camero-Arranz, A., et al. 2010, ApJ, 708, 1500

[12] Campbell, C.G. 1997, Magnetohydrodynamics in Binary Stars (Dordrecht: Kluwer)

[13] Chakrabarty, D., et al. 1997, ApJ, 481, L101

[14] Dai, H.-L., Li, X.-D. 2006, A\&A, 451, 581 
[15] D'Angelo, C.R., \& Spruit, H.C. 2010, MNRAS, 406, 1208

[16] D'Angelo, C., Spruit, H.C. 2011, MNRAS, 416, 893

[17] D’Angelo, C., Spruit, H.C. 2012, MNRAS, 420, 416

[18] Fender, R. et al. 2004, Nature, 427, 222

[19] Fendt, C., \& Elstner, D. 2000, A\&A, 363, 208

[20] Fendt, C. 2003, Astrophys. Space Sci. 287, 59

[21] Ghosh, P., \& Lamb, F. K. 1979, ApJ, 234, 296

[22] Gonzalez-Galan, A. et al. 2012, A\&A, 537, A66

[23] Goodson, A. P., Winglee, R. M., \& Böhm, K.-H. 1997, ApJ, 489, 199

[24] Hayashi, M. R., Shibata, K., \& Matsumoto, R. 1996, ApJ, 468, L37

[25] Königl, A. 1991, ApJ, 370, L39

[26] Foucart, F., Lai, D. 2011, MNRAS, 412, 2799

[27] Illarionov, A.F., Sunyaev R.A., 1975, A\&A, 39, 185

[28] Königl, A. 1991, ApJ, 370, L39.

[29] Lai, D. 1998, ApJ, 502, 721.

[30] Lai D., 1999, ApJ, 524, 1030

[31] Lai D., 2003, ApJ, 591, L119

[32] Lai, D., Foucart, F., Lin, D.N.C. 2011, MNRAS, 412, 2790

[33] Lai D., \& Zhang, H. 2008, ApJ, 683, 949

[34] Lesur, G., \& Longaretti, P.-Y. 2009, A\&A, 504, 309

[35] Lewin, W., van der Klis, M. 2006, Compact Stellar X-ray Sources (Cambridge U. Press)

[36] Li, J., Wickramasinge, D. T., \& Rüdiger, G. 1996, ApJ, 469, 765.

[37] Lii, P.S., et al. 2013, arXiv:1304.2703

[38] Lipunov, V. M., \& Shakura, N. I. 1980, Sov. Astron. Lett., 6, 14.

[39] Locsei, J.T., Melatos, A. 2004, MNRAS, 354, 591

[40] Long, M., Romanova, M.M., \& Lovelace, R.V.E. 2005, ApJ, 634, 1214

[41] Lovelace, R. V. E., Romanova, M. M., \& Bisnovatyi-Kogan, G. S. 1995, MNRAS, 275, 244.

[42] Lovelace, R. V. E., Romanova, M. M., \& Bisnovatyi-Kogan, G. S. 1999, ApJ, 514, 368

[43] Lovelace R. V. E., Romanova M. M., Ustyugova G. V., Koldoba A. V., 2010, MNRAS, 408, 2083

[44] Lynden-Bell, D., Boily, C. 1994, MNRAS, 267, 146

[45] Matt, S., Goodson, A.P., Winglee, R.M., \& Böhm, K.-H. 2002, ApJ, 574, 232

[46] Matt, S., \& Pudritz, R.E. 2005, ApJ, 632, L135

[47] Migliari, S., et al. 2011, MNRAS, 415, 2407

[48] Miller, K. A., \& Stone, J. M. 1997, ApJ, 489, 890

[49] Perna, R., Bozzo, E., Stella, L. 2006, ApJ, 363

[50] Pfeiffer, H.P., Lai, D. 2004, ApJ, 604, 766

[51] Pringle, J. E., \& Rees, M. J. 1972, A\&A, 21, 1

[52] Romanova, M.M., et al. 2003, ApJ, 595, 1009

[53] Romanova, M.M., Kulkarni, A.K., \& Lovelace, R.V.E. 2008, ApJ, 673, L171

[54] Romanova, M.M., et al. 2009, MNRAS, 399, 1802

[55] Romanova M. M., Ustyugova G. V., Koldoba A. V., Lovelace R. V. E., 2012, MNRAS, 421, 63

[56] Romanova,, M.M. et al. 2013, MNRAS, 430, 699

[57] Shirakawa, A., Lai, D. 2002, ApJ, 564, 361 
Physics at the Magnetospheric Boundary

[58] Shirakawa, A., Lai, D. 2002, ApJ, 565, 1134

[59] Shu, F. H., et al. 1994, ApJ, 429, 781

[60] Shu, F.H., Lizano, S., Galli, D., Cai,, M.J., Mohanty, S. 2008, ApJ, 682, L121

[61] Spruit, H.C., Taam, R.E. 1990, A\&A, 229, 475

[62] Spruit, H. C., \& Taam, R. E. 1993, ApJ, 402, 593

[63] Stella, L., Vietri, M. 1998, ApJ, 492, L59

[64] Terquem, C., Papaloizou, J.C.B. 2000, A\&A, 360, 1031

[65] Uzdensky, D.A. 2004, Ap\&SS, 292, 573

[66] Uzdensky, D., Königl, A., \& Litwin, C. 2002, ApJ, 565, 1191

[67] van Ballegooijen, A. A. 1994, Space Science Rev., 68, 299

[68] van der Klis, M. 2006, in Compact Stellar X-ray Sources, eds. W. Lewin, and M. van der Klis (Cambridge U. Press)

[69] Wang, Y.-M. 1987, A\&A, 183, 257

[70] Warner, B. 2004, PASP, 116, 115

[71] Zanni C., Ferreira J., 2013, A\&A, 550, A99 
